Pacific Journal of Mathematics

SEMI-GROUPS OF LOCAL LIPSCHITZIANS IN A BANACH

J. T. CHAMBERS AND SHINNOSUKE CHA 


\section{SEMI-GROUPS OF LOCAL LIPSCHITZIANS IN A BANACH SPACE}

\section{J. T. Chambers AND S. OHARU}

The purpose of this paper is to construct a nonlinear semi-group determined by a given (multi-valued) nonlinear operator $A$ in a Banach space $X$, and to investigate the differentiability of this semi-group. The semi-group treated in this paper is the semigroup $\{T(t) ; t \geqq 0\}$ of nonlinear operators in $X$ such that for each $\tau>0,\{T(t) ; 0 \leqq t \leqq \tau\}$ is equi-Lipschitz continuous on bounded sets. In order that an operator $A$ in $X$ determine such a semi-group $\{T(t) ; t \geqq 0\}$ on $D(A)$ with $(d / d t) T(t) x \in A T(t) x$ for almost all $t \geqq 0$ and $x \in D(A)$, it is required that $X$ have a uniformly convex dual, $\mathbf{A}$ be dissipative in a local sense, $I-\lambda A, \lambda$ positive and small, satisfy a range condition and an injectiveness condition, and finally the family of operators $(I-\lambda A)^{-n}, n=$ $1,2,3, \cdots$ be locally equi-bounded.

Let $X$ be a Banach space and $S$ a subset of $X$, and let $\{T(t)$; $t \geqq[0\}$ be a family of nonlinear operators from $S$ into itself satisfying the following conditions: $s \geqq 0$.

(i) $T(0)=I$ (the identity) and $T(t+s)=T(t) T(s)$ on $S$ for $t$,

(ii) For $x \in S, T(t) x$ is strongly continuous in $t \geqq 0$. Then the family $\{T(t) ; t \geqq 0\}$ is called a semi-group on $S$. The infinitesimal generator $A_{0}$ of the semi-group $\{T(t) ; t \geqq 0\}$ is defined by $A_{0} x=\lim _{h \rightarrow+0} h^{-1}\{T(h) x-x\}$ and the weak infinitesimal generator $A^{\prime}$ by $A^{\prime} x=w$ - $\lim _{h \rightarrow+0} h^{-1}\{T(h) x-x\}$, if the right sides exist, the notation " $w$-lim" means the weak limit in $X$.

An operator $A$ in $X$ is called a $D$-operator if for every bounded set $B$ in $X$ there exists a number $\omega_{B} \geqq 0$ such that

$$
r e<x^{\prime}-y^{\prime}, f>\leqq \omega_{B}\|x-y\|^{2} \text { for } x, y \in B \cap D(A), x^{\prime} \in A x, y^{\prime} \in A y
$$

and some $f \in F(x-y)$, where $F$ denotes the duality mapping of $X$.

Our discussion requires that $X$ have a uniformly convex dual. Then, if $A$ is a $D$-operator satisfying some additional conditions, we obtain a semi-group $\{T(t) ; t \geqq 0\}$ on $\overline{D(A)}$ such that

$$
T(t) x=\lim _{\lambda \rightarrow+0}(I-\lambda A)^{-[t / \lambda]} x,
$$

and the convergence is uniform with respect to $t$ in every finite interval;

(B) for every bounded set $B$ in $D(A)$ and $\tau>0$, there exists a number 
$\omega_{B, \tau} \geqq 0$ such that $\|T(t) x-T(t) y\| \leqq e^{\omega_{B, \tau}}\|x-y\|$ for $x, y \in B$ and $t \in[0, \tau]$.

The additional conditions on $A$ are stated roughly as follows:

(1) The operator $(I-\lambda A)^{-1}$ must exist as a single-valued operator with domain $R(I-\lambda A)$, the range of $I-\lambda A$, for $\lambda$ small; this is condition (I) of the paper.

(2) In order that the iterations of $(I-\lambda A)^{-1}$ be meaningful on $D(A)$, it is required that the range of $I-\lambda A$ contain $D(A)$; this is condition $(R)$.

(3) The operators $(I-\lambda A)^{-k}, k=1,2,3, \cdots$ must map bounded sets into bounded sets; this is the idea behind condition $(E)$.

We note that if $A$ is a dissipative operator, i.e., $\omega_{B} \equiv 0$ for every bounded set $B$ in $X$, then (1) and (3) are satisfied.

Concerning the differentiability of the semi-group constructed we obtain, among other results, the following. If $A$ is a $D$-operator satisfying $(I),(R)$ and $(E)$ and is maximal on $\overline{D(A)}$ in the sense explained in $\S 1$, then there exists a uniquely determined semi-group $\{T(t) ; t \geqq 0\}$ on $\overline{D(A)}$ such that for each $x \in D(A)(d / d t) T(t) x \in A T(t) x$ at almost all $t \geqq 0$.

Finally, we remark that for the Cauchy problem

$$
(d / d t) u(t) \in A u(t), u(0)=x,
$$

where $A$ is a $D$-operator in $X$ satisfying $(I),(R)$ and $(E)$, we can construct the semi-group solution using the convergence $(A)$. And conversely, in a reflexive Banach space, if $A_{0}$ is the infinitesimal generator of a semi-group $\{T(t) ; t \geqq 0\}$ satisfying $(B)$, then $A_{0}$ is a $D$-operator in $X$ and for $x \in D\left(A_{0}\right), T(t) x$ is a solution of the Cauchy problem formulated for the operator $A_{0}$.

Section 1 deals with the notion of a $D$-operator and some of its properties. Section 2 concerns the abstract Cauchy problem. Section 3 contains the construction of the semi-group determined by the $D$-operator $A$. Finally, in Section 4, the question of the differentiability of the constructed semi-group is discussed.

The authors want to express their deep gratitude to Professor I. Miyadera for his many valuable suggestions.

o. Preliminaries. In this section we introduce some of the basic notions which are used in this paper.

Throughout this paper $X$ denotes a Banach space. Let $A$ be a multi-valued operator in $X$, that is, $A$ assigns to each $x \in X$ a subset $A x$ of $X$. $A x$ may be empty for some $x \in X$. The domain of $A$, $D(A)$, is the set of all $x \in X$ such that $A x \neq \varnothing$; the range of $A, R(A)$, is the set $\bigcup_{x \in X} A x$. We write $A S$ (or $A(S)$ ) for $\bigcup_{x \in S} A x, S \subset X$. 
Note that a single-valued operator is a special case of a multi-valued operator in which $A x, x \in D(A)$, denotes the value of $A$ at $x$ or the singleton set consisting of this element, and $A x$ is the empty set if $x \notin D(A)$.

For subsets $S_{1}, S_{2} \subset X, S_{1}+S_{2}$ denotes the set $\left\{x+y ; x \in S_{1}, y \in S_{2}\right\}$ where $S_{1}+S_{2}=\varnothing$ if $S_{1}=\varnothing$ or $S_{2}=\varnothing$. For a scalar $\lambda$ and $S \subset X$, $\lambda S$ denotes the set $\{\lambda x ; x \in S\}$, and we write $y+S$ for $\{y\}+S$.

Accordingly, for two operators $A$ and $B$ in $X$, we define the sum $A+B$ in $X$ by $(A+B) x=A x+B x, D(A+B)=D(A) \cap D(B)$; the scalar multiplication $\lambda A$ in $X$ by $(\lambda A) x=\lambda A x, D(\lambda A)=D(A)$; and the product $A B$ in $X$ by $(A B) x=A(B x), D(A B) \subset D(B)$. We write $\gamma+\lambda A$ for the operator $\gamma I+\lambda A$, where $I$ denotes the identity operator in $X$. For any positive integer $k$, we define the iteration $A^{k}$ in $X$ by $A^{k} x=A\left(A^{k-1} x\right)$, where $A^{0} \equiv I$ and $D\left(A^{k}\right) \subset D(A)$.

Let $A, \widetilde{A}$ be two operators in $X . \widetilde{A}$ is an extension of $A$, and $A$ is a restriction of $\widetilde{A}$ (denoted $\widetilde{A} \supset A, A \subset \widetilde{A}$ ), if $A x \subset \widetilde{A} x$ for each $x \in X$, thus $D(A) \subset D(\widetilde{A})$. If $S \subset X$, then by a restriction of $A$ to $S$, $\left.A\right|_{s}$, we mean the operator such that $D\left(\left.A\right|_{s}\right)=D(A) \cap S$ and $\left.A\right|_{S} x=$ $A x$ if $x \in S$.

If $S \subset X$, we denote the closure of $S$ in $X$ by $\bar{S}$. Let $A$ be an operator in $X$, then $B$ is called the closure of $A$, if $G(B)=\overline{G(A)}$, where $G(\cdot)$ denotes the graph of the operator. We write $B=\bar{A}$.

Let $X^{*}$ be the dual space of $X$. We denote by $\langle x, f\rangle$ the pairing between $x \in X$ and $f \in X^{*}$. The duality mapping $F$ of $X$ is the mapping from $X$ into $X^{*}$ defined by

$$
F(x)=\left\{f \in X^{*} ; \text { re }\langle x, f\rangle=\|x\|^{2}=\|f\|^{2}\right\}
$$

for $x \in X$. If $X^{*}$ is uniformly convex, then $F$ is single-valued and uniformly continuous on bounded sets [4; Lemma 1.2].

We now state some standard definitions and collect some wellknown results.

Definition 1. An operator $A$ in $X$ is said to be dissipative if for each $x, y \in D(A)$ and $x^{\prime} \in A x, y^{\prime} \in A y$, there exists an $f \in F(x-y)$ such that $r e\left\langle x^{\prime}-y^{\prime}, f\right\rangle \leqq 0$. $A$ is said to be an $m$-dissipative operator in $X$, if it is a dissipative operator in $X$ and $R\left(I-\lambda_{0} A\right)=X$ for some $\lambda_{0}>0$. Let $S \subset X$ and $A$ be a dissipative operator in $X$, if every dissipative extension of $A$ coincides on $S$ with $A$, then $A$ is said to be a maximal dissipative operator on $S$.

An $m$-dissipative operator $A$ is maximal dissipative on $D(A)$. If $X^{*}$ is strictly convex and $A$ is a maximal dissipative opetator on $S$, then $A x$ is closed and convex for $x \in S$. If $A$ is an $m$-dissipative operator, then $R(I-\lambda A)=X$ for all $\lambda>0$ ([9; Lemma 4]). 
Definition 2. An operator $A$ in $X$ is said to be demi-closed if the following condition holds: if $\left\{x_{n}\right\} \subset D(A), x_{n} \rightarrow x \in X$ (strong convergence) and if $y_{n} \in A x_{n}$, such that $y_{n} \rightarrow y \in X$ (weak convergence) implies that $x \in D(A)$ and $y \in A x$.

A demi-closed operator is closed. If $X^{*}$ is uniformly convex and $A$ is maximal dissipative on $\overline{D(A)}$, then $A$ is demi-closed ([5; Lemma $3.7])$.

Definition 3. Let $A$ be an operator in $X$. The operator $A^{0}$ defined by $A^{0} x=\{y \in A x ;\|y\|=\inf [\|u\| ; u \in A x]\}$ is called the canonical restriction of $A$.

If $X^{*}$ is uniformly convex and $A$ is an $m$-dissipative operator, then $D\left(A^{0}\right)=D(A)$ and $A^{0} x$ is a non-empty closed convex set for $x \in D(A)$. If $X$ and $X^{*}$ are uniformly convex and $A x$ is closed and convex for $x \in X$, then $A^{0}$ is single-valued and $D\left(A^{0}\right)=D(A)$ ([5; Lemma 3.10]).

Finally, we list some notations which are used in this paper.

(1) Let $\left\{x_{n}\right\}$ be a sequence in $X$, then " $x_{n} \rightarrow x$ ", means that $x_{n}$ converges to $x$ in the norm topology, whereas, " $x_{n} \rightarrow x$ ", means that $x_{n}$ converges to $x$ in the weak topology.

(2) Let $G$ be a single-valued operator in $X$ and $B \subset X$, then by $\|G\|_{\text {Lip (B) }}$, we mean the smallest Lipschitz constant for $G$ on $B \cap D(G)$.

(3) We write $J_{\lambda}$ for the resolvent $(I-\lambda A)^{-1}$ if it is well-defined and $R_{\lambda}$ for the range $R(I-\lambda A)=\{x-\lambda y ; x \in D(A), y \in A x\}$.

(4) Let $K \subset X$. Then $c o K$ denotes the convex hull of $K$ and $\overline{c o} K$, the convex closure of $K$.

(5) For any nonempty set $S \subset X$, we write

$$
\||| S \mid\|=\inf \{\|x\| ; x \in S\} \text {. }
$$

Thus for any operator $A,\|A x\|$ is defined for $x \in D(A)$

1. D-operators. In this section we introduce the notion of a $D$ operator and establish some of its properties.

Let $X$ be a Banach space and $A$ an operator in $X$. If for every bounded set $B \subset X$ there exists a nonnegative number $\omega_{B}$ such that

$$
r e<x^{\prime}-y^{\prime}, f>\leqq \omega_{B}\|x-y\|^{2}
$$


for $x, y \in B \cap D(A), x^{\prime} \in A x, y^{\prime} \in A y$ and for some $f \in F^{\prime}(x-y)$, then $A$ is called a $D$-operator.

Put $B_{n}=\{x \in X ;\|x\|<n\}, n=1,2,3, \cdots$ If there exists a sequence $\left\{\omega_{n}\right\}$ of nonnegative numbers such that

$$
r e\left\langle x^{\prime}-y^{\prime}, f\right\rangle \leqq \omega_{n}\|x-y\|^{2}
$$

for $x, y \in B_{n} \cap D(A), x^{\prime} \in A x, y^{\prime} \in A y$ and for some $f \in F(x-y), n=$ $1,2,3, \cdots$, then $A$ is a $D$-operator. If such a sequence is identically zero, then $A$ is a dissipative operator. Note that if $A$ is a $D$-operator, then $\left.\left(A-\omega_{n}\right)\right|_{B_{n}}$ is a dissipative operator on $B_{n}$.

The next lemma by Kato [4; Lemma 1.1] gives a basic property of dissipative operators.

Proposition 1.1. (Kato) Let $x, y \in X$. Then there is a $\lambda_{0}>0$ such that $\|x\| \leqq\|x-\lambda y\|$ for $\lambda \in\left(0, \lambda_{0}\right)$ if and only if there is an $f \in F(x)$ such that $r e\langle y, f\rangle \leqq 0$.

Let $A$ be a $D$-operator in $X$, then for every bounded set $B \subset D(A)$, we have that $(I-\lambda A) x \cap(I-\lambda A) y=\varnothing$ for $x, y \in B$, if $x \neq y$ and $\lambda \in\left(0,1 / \omega_{B}\right)$. In fact, for $x^{\prime} \in A x, y^{\prime} \in A y$, and some $f \in F(x-y)$ we have that

$$
\begin{aligned}
\left\|\left(x-\lambda x^{\prime}\right)-\left(y-\lambda y^{\prime}\right)\right\|\|x-y\| & \geqq r e\left\langle\left(x-\lambda x^{\prime}\right)-\left(y-\lambda y^{\prime}\right), f\right\rangle \\
& \geqq\left(1-\lambda \omega_{B}\right)\|x-y\|^{2} .
\end{aligned}
$$

Hence, we have $\left\|\left(x-\lambda x^{\prime}\right)-\left(y-\lambda y^{\prime}\right)\right\| \geqq\left(1-\lambda \omega_{B}\right)\|x-y\|, \quad$ so, $\left.(I-\lambda A)\right|_{B}$ has a Lipschitz continuous inverse and

$$
\left\|\left(I-\left.\lambda A\right|_{B}\right)^{-1}\right\|_{\operatorname{Lip}((I-\lambda A) B)} \leqq\left(1-\lambda \omega_{B}\right)^{-1} \text { for } \lambda \in\left(0,1 / \omega_{B}\right) .
$$

However, in general, $(I-\lambda A)^{-1}$ is not a single-valued operator. For example, take $X$ to be the real line and $A x$, the function $x \sin x . A$ is a $D$-operator, in fact, for the bounded set $[-M, M]$ we may take $\omega_{[-M, M]}$ to be $1+M$. And

$\|\left.\left(I-\left.\lambda A\right|_{[-M, M]}\right)^{-1}\right|_{\operatorname{Lip}((I-\lambda A)[-M, M]} \leqq(1-\lambda(1+M))^{-1}$ for $\lambda \in(0,1 /(1+M))$.

But $R(I-\lambda A)=X$ for $\lambda>0$, and $(I-\lambda A)^{-1}$ can not defined as a single-valued operator on $X$, no matter how small we restrict $\lambda>0$. Hence, we make an additional assumption on the operator $A$ :

(I) $\quad(I-\lambda A) x \cap(I-\lambda A) y=\varnothing$ for $x, y \in X$ if $x \neq y$ and $\lambda \in\left(0, \lambda_{0}\right)$.

Condition (I) guarantees the existence of the resolvent $J_{\lambda} \equiv(I-\lambda A)^{-1}$ for $\lambda \in\left(0, \lambda_{0}\right)$ as a single-valued operator with $D\left(J_{\lambda}\right)=R(I-\lambda A)$. 
corresponds to the assumption that $I-\lambda A$ is injective, if we are considering single-valued operators.

Definition 1.1. Let $G$ be an operator in $X . G$ is said to be locally bounded if $G$ maps bounded sets into bounded sets.

DeFINITION 1.2. Let $\left\{G_{\gamma}\right\}, \gamma \in \Gamma$, be a family of operators in $X$. $\left\{G_{\gamma}\right\}, \gamma \in \Gamma$, is said to be locally equi-bounded, if for every bounded set $B, \cup_{\gamma \in \Gamma} G_{\gamma}(B)$ is a bounded set.

Proposition 1.2. Let $A$ be a D-operator in $X$ satisfying (I). If $\left\{J_{\lambda} ; \lambda \in\left(0, \lambda_{0}\right)\right\}$ is locally equibounded, then for every bounded set $B \subset X$ there exists a number $\tilde{\omega}_{B} \geqq 0$ such that

$$
\left\|J_{\lambda}\right\|_{\mathrm{Lip}(B)} \leqq\left(1-\lambda \tilde{\omega}_{B}\right)^{-1} \quad \text { for } \lambda \in\left(0, \min \left\{\lambda_{0}, \frac{1}{\tilde{\omega}_{B}}\right\}\right) .
$$

Proof. Let $B$ be any bounded set in $X$, then $B_{1} \equiv \bigcup_{\lambda \in\left(0 \lambda_{0}\right)} J_{\lambda}(B)$ is a bounded set and $B_{1} \subset D(A)$. Hence, there exists a number $\tilde{\omega}_{B} \geqq 0$ such that $\left(1-\lambda \tilde{\omega}_{B}\right)\|x-y\| \leqq\left\|\left(x-\lambda x^{\prime}\right)-\left(y-\lambda y^{\prime}\right)\right\|$ for $x, y \in B_{1}$, $x^{\prime} \in A x, y^{\prime} \in A y$ and $\lambda \in\left(0, \min \left\{\lambda_{0}, 1 / \tilde{\omega}_{B}\right\}\right)$. Thus, if $u, v \in B \cap R_{\lambda}$, then $J_{\lambda} u, J_{\lambda} v \in B_{1}$ and

$$
\left(1-\lambda \tilde{\omega}_{B}\right)\left\|J_{\lambda} u-J_{\lambda} v\right\| \leqq\|u-v\| \quad \text { for } \lambda \in\left(0, \min \left\{\lambda_{0}, \frac{1}{\tilde{\omega}_{B}}\right\}\right) .
$$

In the next proposition we impose two additional conditions on the operator $A$, which are essential to the construction of the semigroup in this paper.

Proposition 1.3. Let $A$ be a D-operator in $X$ satisfying (I). If

$$
R(I-\lambda A) \supset D(A) \quad \text { for } \lambda \in\left(0, \lambda_{0}\right),
$$

and

$$
\left\{J_{2}^{n} ; \lambda \in\left(0, \lambda_{0}\right), n \lambda \in[0, \tau]\right\}
$$

is locally equi-bounded for any $\tau>0$, then for every bounded set $B \subset X$ and $\tau>0$, there exists a number $\omega_{B, \tau} \geqq 0$ such that

$$
\left\|J_{\lambda}^{n}\right\|_{\operatorname{Lip}(B)} \leqq\left(1-\lambda \omega_{B, \tau}\right)^{-n}
$$

for $\lambda \in\left(0, \min \left\{\lambda_{0}, 1 / \omega_{B, \tau}\right\}\right)$ and $n \lambda \in[0, \tau]$.

Proof. Let $B$ be any bounded set in $X$ and $\tau>0$. Set $B_{1}=$ $\bigcup_{\substack{\lambda \in\left(0, \lambda_{0}\right) \\ n \in \in[0, \tau]}} J_{\lambda}^{n}(B)$, then $B_{1} \cup B$ is a bounded set and so there exists a 
number $\omega_{B: \tau} \geqq 0$ such that

$$
\left\|J_{\lambda}\right\|_{\mathrm{Lip}\left(B_{1} \cup B\right)} \leqq\left(1-\lambda \omega_{B, \tau}\right)^{-1} \quad \text { for } \lambda \in\left(0, \min \left\{\lambda_{0}, \frac{1}{\omega_{B, \tau}}\right\}\right) .
$$

Thus, if $u, v \in B \cap R_{\lambda}$, then $J_{\lambda}^{n-1} u, J_{\lambda}^{n-1} v \in B_{1} \cup B$, provided $n \lambda \in[0, \tau]$. So that $\left\|J_{\lambda}^{n} u-J_{\lambda}^{n} v\right\| \leqq\left(1-\lambda \omega_{B, \tau}\right)^{-1}\left\|J_{\lambda}^{n-1} u-J_{\lambda}^{n-1} v\right\| \leqq\left(1-\lambda \omega_{B, \tau}\right)^{-n}\|u-v\|$ for $\lambda \in\left(0, \min \left\{\lambda_{0}, 1 / \omega_{B, \tau}\right\}\right)$.

The next proposition gives some sufficient conditions for $(E)$.

Proposition 1.4. Let $A$ be a D-operator satisfying (I).

(a) If there exist nonnegative numbers $M$ and $N$ such that

$$
\left\|J_{\lambda} x\right\| \leqq(1+\lambda M)\|x\|+\lambda N \quad \text { for } \lambda \in\left(0, \lambda_{0}\right) \text { and } x \in R_{\lambda},
$$

then $(E)$ holds.

(E) holds.

(b) If $A$ is single-valued and $\sup \{\|A x\| ; x \in D(A)\}<+\infty$, then

Proof. (a) Let $B$ be any bounded set in $X$ and $x \in B \cap R_{\lambda}$, $\lambda \in\left(0, \lambda_{0}\right)$. Then, it is easy to see that

$$
\left\|J_{\lambda}^{n} x\right\| \leqq(1+M \lambda)^{n}(\|x\|+n \lambda N) \leqq e^{M n \lambda}(\sup \|x\|+n \lambda N),
$$

which is bounded for $\lambda \in\left(0, \lambda_{0}\right)$ and $n \lambda \in[0, \tau]$.

(b) Take $x \in R_{\lambda}$, then $\left\|J_{\lambda} x\right\| \leqq\|x\|+\lambda\left\|A J_{i} x\right\|$. Put

$$
N=\sup _{x \in D(A)}\|A x\| \text {, }
$$

then $\left\|J_{\lambda} x\right\| \leqq\|x\|+\lambda N$. Now apply (a), note that in this case $M=0$.

We now wish to introduce a notion of maximal $D$-operator. Given a sequence of nondecreasing nonnegative numbers $\left\{\omega_{n}\right\}$, we consider the family of $D$-operators, $\mathscr{F}\left\{\omega_{n}\right\}$, consisting of all $D$-operators $A$ in $X$ such that there exist numbers $\omega_{B_{n}}(A) \leqq \omega_{n}, \quad n=1,2,3, \cdots$ with

$$
r e\left\langle x^{\prime}-y^{\prime}, f\right\rangle \leqq \omega_{B_{n}}(A)\|x-y\|^{2}
$$

for $x, y \in D(A) \cap B_{n}, x^{\prime} \in A x, y^{\prime} \in A y$ and some $f \in F(x-y), n=1,2$, $3, \cdots$. Recall that $B_{n}$ denotes the open ball with radius $n$ and center 0 in $X$. Note that if $A$ is a $D$-operator, then there exists a sequence $\left\{\omega_{n}\right\}$ such that $A \in \mathscr{F}\left\{\omega_{n}\right\}$.

Definition 1.3. If $A \in \mathscr{F}\left\{\omega_{n}\right\}$, then $A$ is called a $\left(D,\left\{\omega_{n}\right\}\right)$-operator. Let $S \subset X$ and $A$ be a $\left(D,\left\{\omega_{n}\right\}\right)$-operator in $X$. If every 
( $\left.D,\left\{\omega_{n}\right\}\right)$-extension of $A$ coincides on $S$ with $A$, then $A$ is said to be a maximal $\left(D,\left\{\omega_{n}\right\}\right)$-operator on $S$.

Proposition 1.5. If $A$ is a $\left(D,\left\{\omega_{n}\right\}\right)$-operator in $X$ and $S \subset X$, then there exists a maximal $\left(D,\left\{\omega_{n}\right\}\right)$-operator $\tilde{A}$ on $S$ such that $\left.\left.\widetilde{A}\right|_{s} \supset A\right|_{s}$.

\section{Proof. Apply Zorn's Lemma.}

We now show that if $A$ is a maximal $\left(D,\left\{\omega_{n}\right\}\right)$-operator on $\overline{D(A)}$ and furthermore if $X^{*}$ is uniformly convex, then $A$ is demi-closed and $A x$ is closed and convex. The uniform convexity of $X^{*}$ gives the above properties which are essential in establishing the facts concerning the differentiability of the semi-groups constructed in this paper.

Proposition 1.6. Let $X^{*}$ be uniformly convex. If $A$ is a maximal $\left(D,\left(\omega_{n}\right)\right\}$-operator on $\overline{D(A)}$, then

(a) $A$ is demi-closed,

(b) $A x$ is closed convex.

Proof. (a) Let $\left\{x_{k}\right\}$ be a sequence such that $\left\{x_{k}\right\} \subset D(A), x_{k} \rightarrow x_{0} \in X$ and $A x_{k} \ni y_{k} \rightarrow y$. We must show that $x_{0} \in D(A)$ and $y \in A x_{0}$. Define $\widetilde{A} w=A w$ if $w \neq x_{0}$ and $A w \cup\{y\}$ if $w=x_{0}$. Then $\widetilde{A} \supset A$ and $D(\widetilde{A}) \subset \overline{D(A)}$. It is easy to see that $\widetilde{A}$ is a $\left(D,\left\{\omega_{n}\right\}\right)$-operator, Hence, by the maximality of $A, \widetilde{A}=A$ and so $x_{0} \in D(A)$ with $y \in A x_{0}$.

(b) The same type of argument as in (a) easily establishes (b).

The next proposition states some basic properties of a demi-closed operator.

Proposition 1.7. Let $X$ be a reflexive Banach space and $A$ be a demi-closed operator in $X$. Let $\left\{x_{n}\right\} \subset D(A), x_{n} \rightarrow x_{0} \in X$, and let $\left\{y_{n}\right\}$ be a sequence in $X$ such that $y_{n} \in A x_{n}$ for each $n$. Then:

(a) if $\left\{y_{n}\right\}$ is bounded and $V$ is the set of all weak cluster points of $\left\{y_{n}\right\}$, then $x_{0} \in D(A), V \neq \varnothing$, and $V \subset A x_{0}$; if in particular, $A$ is single-valued, then $y_{n} \rightarrow A x_{0}$;

(b) if furthermore, $X$ and $X^{*}$ are uniformly convex, the canonical restriction $A^{0}$ is single-valued and if lim $\sup \left\|y_{n}\right\| \leqq\left\|A x_{0}\right\|$, then $x_{0} \in D\left(A^{0}\right)$ and $y_{n} \rightarrow A^{0} x_{0}$.

Proof. (a) First, the reflexivity of $X$ and the boundedness of $\left\{y_{n}\right\}$ imply that $V \neq \varnothing$. Let $Y$ be the closed linear manifold determined by $\left\{y_{n}\right\}$. Then $Y$ is a reflexive Banach space and $Y^{*}$ is 
separable. Hence, for each $y \in V$ a subsequence $\left\{y_{n_{i}}\right\}$ can be found such that $y_{n_{i}} \rightarrow y$ in $Y$. Now, any $x^{*} \in X^{*}$ determines a $y^{*} \in Y^{*}$ such that $\left\langle x, x^{*}\right\rangle=\left\langle x, y^{*}\right\rangle$ for $x \in Y$; thus, since $y_{n} \in Y,\left\langle y_{n_{i}}, x^{*}\right\rangle \rightarrow$ $\left\langle y, x^{*}\right\rangle$ for $x^{*} \in X^{*}$, and $y_{n_{i}} \rightarrow y$ in $X$. Since $x_{n_{i}} \rightarrow x_{0}, y_{n_{i}} \in A x_{n_{i}}, y_{n_{i}} \rightarrow y$ and since $A$ is demi-closed, we have that $x_{0} \in D(A)$ and $y \in A x_{0}$. This means that $V \subset A x_{0}$. If in particular, $A$ is single-valued, then $y=$ $A x_{0}$; hence all weak limits of subsequences of $\left\{y_{n}\right\}$ are same and equal to $A x_{0}$. Therefore, it follows that $y_{n} \rightarrow A x_{0}$.

(b) Since $\left\{y_{n}\right\}$ is bounded and $A$ is demi-closed, there is a subsequence $\left\{y_{n_{i}}\right\}$ and a $y \in A x_{0}$ such that $y_{n_{i}} \rightarrow y$. Thus, by assumption, we have

$$
\text { II } A x_{0}\|\mid \leqq\| y\|\leqq \lim \inf \| y_{n_{i}}\|\leqq \lim \sup \| y_{n_{i}}\|\leqq\|\left\|x_{0}\right\| \|
$$

Since $A^{0}$ is single-valued, $y=A^{0} x_{0}$ and $\lim \left\|y_{n_{i}}\right\|=\left\|A^{0} x_{0}\right\|$. But, $X$ is uniformly convex; thus $y_{n_{i}} \rightarrow A^{0} x_{0}$. Therefore, all strong limits of subsequences are same and equal to $A^{0} x_{0}$, and it follows that $y_{n} \rightarrow A^{0} x_{0}$.

Proposition 1.8. Let $X$ and $X^{*}$ be uniformly convex. Let $A$ be a closed D-operator in $X$ satisfying (I), (3.9) (stated in Remark 3.1) and (E). If $\widetilde{A}$ is a maximal $\left(\left(D,\left\{\omega_{n}\right\}\right)\right.$-extension of $A$ on $\overline{D(A)}$ such that

$$
D(\widetilde{A}) \subset\left\{z \in \overline{D(A)} ;\left\|J_{\lambda} z-z\right\|=0(\lambda) \text { as } \lambda \downarrow 0\right\},
$$

then $A^{0}=\widetilde{A}^{0}$.

Proof. First, note that $\widetilde{A}$ is demi-closed, $\widetilde{A} x$ is closed and convex, and so, $\widetilde{A}^{0}$ is single-valued with $D\left(\widetilde{A}^{0}\right)=D(\widetilde{A})$. Take a sequence $\eta_{k} \downarrow 0$ and set $J_{k}=J_{\eta_{k}}$ and $A_{k}=\eta_{k}^{-1}\left[J_{k}-I\right]$. Let $x \in D(\widetilde{A})$, then, since $D\left(A_{k}\right) \supset D(\widetilde{A})$, we see that $\left\|A_{k} x\right\|=\eta_{k}^{-1}\left\|J_{k} x-J_{k}\left(x-\eta_{k} y\right)\right\| \leqq\|\widetilde{A} x\| \|$ $\left(1-\omega_{B} \eta_{k}\right)$ for $y \in \widetilde{A}^{0} x$, where $\omega_{B}$ is a constant associated with the closure $B$ of $\left\{x-\eta_{k} y ; y \in \widetilde{A} x,\|y\|=\|\widetilde{A} x\| \|, k\right.$ sufficiently large $\}$ through the $D$-operator $A$. Since $A_{k} x \in \widetilde{A} J_{k} x$ for $k, A_{k} x \rightarrow \widetilde{A}^{0} x$ as $k \rightarrow+\infty$ by Proposition $1.7(\mathrm{~b})$, for each $x \in D(\widetilde{A})$. Now take $z \in D(\widetilde{A})$. Since $R(I-\lambda A) \supset \overline{D(A)}$ for $\lambda \in\left(0, \lambda_{0}\right)$ by assumption and since $\overline{D(A)} \supset D(\widetilde{A})$, we see that $z \in R\left(I-\eta_{k} A\right)$ for $k$ sufficiently large. Hence, there exist $x_{k} \in D(A)$ and $y_{k} \in A x_{k}$ such that

$$
z=x_{k}-\eta_{k} y_{k}
$$

But, $x_{k}=J_{k} z \rightarrow z$ as $k \rightarrow+\infty$; hence, by the closedness of $A, z \in D(A)$ and $\widetilde{A}^{0} z \in A z$. But $A z \subset \widetilde{A} z$, so that $\widetilde{A}^{0} z \in A^{0} z$. Also, $\left\|A^{0} z\right\| \leqq\left\|\widetilde{A}^{0} z\right\|$. Therefore, $v \in A^{0} z \subset \widetilde{A} z$ implies that $v=\widetilde{A}^{0} z$ because $\widetilde{A}^{0}$ is singlevalued. So, $A^{0}$ is also single-valued. 
Remark 1.1. Brezis and Pazy [1; Theorem 2.1] give the following result. Let $X$ be a Hilbert space and $A$ be a closed dissipative operator such that $R_{\lambda} \supset \overline{c o} D(A)$ for all $\lambda>0$, then $A$ has a unique extension to a maximal dissipative operator $\widetilde{A}$ satisfying $D(\widetilde{A}) \subset \overline{D(A)}$, and, in fact, $D(\widetilde{A})=D(A)$ and $\widetilde{A}^{0}=A^{0}$.

2. Abstract Caucy problem. In this section we discuss the relationship between the abstract Cauchy problem formulated for a $D$-operator and the semi-group generated by such an operator.

The abstract Cauchy problem may be stated as follows:

Given an operator $A$ in $X$ and an element $x \in X$, find a $X$-valued function $u(t ; x)$ on $[0, \infty)$ such that

(i) $u(t ; x)$ is strongly absolutely continuous on every finite interval;

(ii) $u(0 ; x)=x$ and $(d / d t) u(t ; x) \in A u(t ; x)$ for almost all $t$.

We call this the abstract Cauchy problem, $A C P$, formulated to $A$.

Proposition 2.1. Let $A$ be a D-operator in $X$. Then there is at most one solution of the ACP formulated to $A$ with the initial value $x \in D(A)$.

Proof. For $x \in D(A)$, suppose that $u(t ; x)$ and $v(t ; x)$ are solutions of the $A C P$ formulated to $A$. By Kato's lemma [4; Lemma 1.3] we have that

$$
\begin{aligned}
\|u(t ; x)-v(t ; x)\|^{2} & =2 \int_{0}^{t} r e\left\langle\left(\frac{d}{d s}\right) u(s ; x)-\left(\frac{d}{d s}\right) v(s ; x), f(s)\right\rangle d s \\
& \leqq 2 \omega_{x, \tau} \int_{0}^{t}\|u(s ; x)-v(s ; x)\|^{2} d s
\end{aligned}
$$

where $\omega_{x, \tau}$ is a constant associated with the bounded set

$$
B=\{u(t ; x), v(t ; x) ; t \in[0, \tau]\}
$$

through the $D$-operator $A$ and $f(s) \in F(u(s ; x)-v(s ; x))$, and also, note that $(d / d s) u(s ; x) \in A u(s ; x)$ and $(d / d s) v(s ; x) \in A v(s ; x)$ for almost all $s$. Hence, $u(t ; x) \equiv v(t ; x)$ for $t \in[0, \tau]$. Since $\tau$ is arbitrary, $u(t ; x) \equiv v(t ; x)$ for all $t \geqq 0$.

Proposition 2.2. If $A$ is a D-operator in $X$ such that for each $x \in D(A)$, there is a solution $u(t ; x)$ to the $A C P$ formulated to $A$ satisfying the condition that for any sequentially compact set $K \subset D(A)$ and $\tau>0,\{u(t ; x) ; t \in[0, \tau], x \in K\}$ is bounded, then there is a semigroup $\{T(t) ; t \geqq 0\}$ defined on $\overline{D(A)}$ and such that $T(t) x \equiv u(t ; x), x \in D(A)$ and $t \in[0, \tau]$. Conversely, if $X$ is reflexive and $A_{0}$ is the infinitesimal 
generator of a semi-group $\{T(t) ; t \geqq 0\}$ satisfying the condition that for every $\tau>0$ and bounded set $B$ there is a constant $\omega_{B, \tau} \geqq 0$ such that $\|T(t)\|_{\text {Lip }(B)} \leqq \exp \left(\omega_{B, \tau} t\right), t \in[0, \tau]$, then $A_{0}$ is a D-operator in $X$ and for each $x \in D\left(A_{0}\right), T(t) x$ is a solution of the ACP formulated to $A_{0}$.

Proof. Take $x \in D(A)$ and $\tau>0$, and put $T(t) x \equiv u(t ; x)$, $t \in[0, \tau]$. Since $u(t ; x) \in D(A)$ for almost all $t \in[0, \tau]$ and $u(t ; x)$ is strongly continuous, $u(t ; x) \in \overline{D(A)}$, i.e., $T(t) x \in \overline{D(A)}$ for all $t \in[0, \tau]$. Hence, $T(t)$ maps $D(A)$ into $\overline{D(A)}$. By Kato's lemma, for $x, y \in K$, a compact set, we have that

$$
\begin{aligned}
\|T(t) x-T(t) y\|^{2}-\|x-y\|^{2} & =2 \int_{0}^{t} r e\left\langle\left(\frac{d}{d s}\right) T(s) x-\left(\frac{d}{d s}\right) T(s) y, f(s)\right\rangle d s \\
& \leqq 2 \omega_{K, \tau} \int_{0}^{t}\|T(s) x-T(s) y\|^{2} d s,
\end{aligned}
$$

where $\omega_{K, \tau}$ is a number associated with the bounded set

$$
\{T(t) x ; t \in[0, \tau], x \in K\}
$$

and $f(s) \in F(T(s) x-T(s) y)$, and also, note that $(d / d s) T(s) x \in A T(s) x$ and $(d / d s) T(s) y \in A T(s) y$ for almost all $s$. Therefore,

$$
\|T(t) x-T(t) y\| \leqq \exp \left(\omega_{K, \tau} t\right)\|x-y\|, x, y \in K, t \in[0, \tau] .
$$

Now, take $z \in \overline{D(A)}$, then there exists a sequence $\left\{x_{n}\right\} \subset D(A)$ such that $x_{n} \rightarrow z$, and so, $\left\|T(t) x_{n}-T(t) x_{m}\right\| \leqq \exp \left(\omega_{K, \tau} t\right)\left\|x_{n}-x_{m}\right\|$ where $K=\left\{x_{n}\right\}$. Hence, define $T(t) z=\lim _{n \rightarrow \infty} T(t) x_{n}$, thus $T(t)$ maps $\overline{D(A)}$ into itself. The semi-group property follows from the uniqueness of the solution of the $A C P$. Conversely, take any bounded set $B$ in $D\left(A_{0}\right)$, then for $x, y \in B$

$$
r e\left\langle h^{-1}(T(h) x-x)-h^{-1}(T(h) y-y), f\right\rangle \leqq h^{-1}\left(\exp \left(\omega_{B, \tau} h\right)-1\right)\|x-y\|^{2}
$$

where $h \in[0, \tau]$ and $f \in F(x-y)$. Letting $h \rightarrow+0$, we have that

$$
r e\left\langle A_{0} x-A_{0} y, f\right\rangle \leqq \omega_{B, \tau}\|x-y\|^{2},
$$

so $A_{0}$ is a $D$-operator. Let $x \in D\left(A_{0}\right)$, then

$$
\sup \left\{h^{-1}\|T(h) x-x\| ; 0<h \leqq 1\right\}=M<+\infty
$$

and $\|T(t+h) x-T(t) x\| \leqq M e x p\left(\omega_{B, \tau} t\right) h$ for $t \in[0, \tau], h \in(0,1]$ and $B=\{T(h) x ; h \in(0,1]\}$. Thus, $T(t) x$ is strongly absolutely continuous on every finite interval. Since $x$ is reflexive, $T(t) x$ is strongly differentiable for almost all $t \in[0, \tau]$ and $(d / d t) T(t)=A_{0} T(t) x$ for almost all $t \in[0, \tau]$. Therefore, $T(t) x$ is a solution of the $A C P$ formulated 
to $A_{0}$.

Combining the properties mentioned above we have the following:

Proposition 2.3. Let $A$ be a D-operator in $x$. Then there is at most one semi-group $\{T(t) ; t \geqq 0\}$ on $\overline{D(A)}$ such that for each $x \in D(A)$, $T(t) x$ is a solution of the ACP formulated to $A$.

3. Construction of the semi-groups. In this section, we construct the semigroup determined by a $D$-operator $A$ which satisfies conditions (I), (R) and (E).

Throughout, it is assumed that $X$ has a uniformly convex dual.

Lemma 3.1. Let $A$ be a D-operator in $X$ satisfying $(I),(R)$ and $(E)$. If $x \in D(A)$ and $\tau>0$, then

$$
y(t ; x)=\lim _{\lambda \rightarrow+0}(I-\lambda A)^{-[t / \lambda]} x
$$

exists uniformly for $t \in[0, \tau]$.

Proof. Set $J_{\lambda}=(I-\lambda A)^{-1}$ and $A_{\lambda}=\lambda^{-1}\left(J_{\lambda}-I\right), \lambda \in\left(0, \lambda_{0}\right)$. Let $x \in D(A)$ and $\tau>0$. Set

$$
\begin{aligned}
B_{x, \tau} & =\left\{J_{h}^{m} x ; h \in\left(0, \lambda_{0}\right), m h \in[0, \tau]\right\} \cup\left\{x-h y ; h \in\left(0, \lambda_{0}\right), y \in A x,\|y\|\right. \\
& \leqq\|A x\|+1\},
\end{aligned}
$$

then $B_{x, \tau}$ is a bounded set by $(E)$. Let $\omega_{B_{x, \tau}}$ be a number associated with this bounded set in the sense of Proposition 1.3. Then we have that

$$
\begin{aligned}
\left\|A_{h} J_{h}^{m-1} x\right\| & =h^{-1}\left\|J_{h}^{m} x-J_{h}^{m-1} x\right\|=h^{-1}\left\|J_{h}^{m} x-J_{h}^{m}(x-h y)\right\| \\
& \leqq\left(1-h \omega_{B x, \tau}\right)^{-m}\|y\|
\end{aligned}
$$

for $y \in A x$ with $\|y\| \leqq\|A x\| \mid+1$. Hence, a positive number $C_{x, \tau}$ can be found such that

$$
\left\|A_{h} J_{h}^{m-1} x\right\| \leqq\left(1-h \omega_{B_{x, \tau}}\right)^{-m}|\|A x\|| \leqq C_{x, \tau},
$$

for $h$ sufficiently small and $m h \in[0, \tau]$. Now, assume that $\lambda n \leqq h$ and $h m \leqq \tau$, where $h \in\left(0, \lambda_{0}\right)$ and $m, n$ are integers. And let $k \leqq m$. Since

$$
J_{\lambda}^{n k} x-J_{\lambda}^{n(k-1)} x=\lambda \sum_{p=0}^{n-1} A_{\lambda} J_{\lambda}^{p} J_{\lambda}^{n(k-1)} x
$$

we have 


$$
\begin{aligned}
& \left(J_{\lambda}^{n k} x-J_{\lambda}^{n(k-1)} x\right)-\left(J_{h}^{k} x-J_{h}^{k-1} x\right) \\
= & \lambda \sum_{p=0}^{n-1}\left\{A_{\lambda} J_{\lambda}^{n(k-1)+p} x-A_{h} J_{h}^{k-1} x\right\}+(n \lambda-h) A_{h} J_{h}^{k-1} x .
\end{aligned}
$$

Thus, we can write

$$
\begin{aligned}
& \left\langle\left(J_{\lambda}^{n k} x-J_{\lambda}^{n(k-1)} x\right)-\left(J_{h}^{k} x-J_{h}^{k-1} x\right), F\left(J_{\lambda}^{n k} x-J_{h}^{k} x\right)\right\rangle \\
= & \lambda \sum_{p=0}^{n-1}\left\langle A_{\lambda} J_{\lambda}^{n(k-1)+p} x-A_{h} J_{h}^{l k-1} x, F\left(J_{\lambda}^{n(k-1)+p+1} x-J_{h}^{k} x\right)\right\rangle \\
& +\lambda \sum_{p=0}^{n-1}\left\langle A_{\lambda} J_{\lambda}^{n(k-1)+p} x-A_{h} J_{h}^{k-1} x, F\left(J_{\lambda}^{n k} x-J_{h}^{k} x\right)\right. \\
& \left.-F\left(J_{\lambda}^{n(k-1)+p+1} x-J_{h}^{k} x\right)\right\rangle \\
& +(n \lambda-h)\left\langle A_{h} J_{h}^{k-1} x, F\left(J_{\lambda}^{n k} x-J_{h}^{k} x\right)\right\rangle \equiv I_{1}+I_{2}+I_{3} .
\end{aligned}
$$

We now estimate each term. Since $A$ is a $D$-operator and $B_{x, \tau}$ is a bounded set, $I_{1} \leqq \lambda \sum_{p=0}^{n-1} \omega_{B_{x, \tau}}\left\|J_{\lambda}^{n(k-1)+p+1} x-J_{h}^{k} x\right\|^{2}$. Since

$$
\begin{aligned}
& \left\|J_{\lambda}^{n(k-1)+p+1} x-J_{\lambda}^{n k} x\right\| \leqq \sum_{j=n(k-1)+p+1}^{n k-1}\left\|J_{\lambda}^{j} x-J_{\lambda}^{j+1} x\right\| \\
\leqq & \lambda \sum_{j=n(k-1)+1}^{n k-1}\left\|A_{\lambda} J_{\lambda}^{j} x\right\| \leqq \lambda\left(1-\lambda \omega_{B_{x}, \tau}\right)^{-n k+1} n\|A x\| \\
\leqq & C_{x, \tau} n \lambda \leqq C_{x, \tau} h
\end{aligned}
$$

we have

$$
I_{1} \leqq \omega_{B_{x, \tau}} h\left\|J_{\lambda}^{n k} x-J_{h}^{k} x\right\|^{2}+\text { const }(x, \tau) h,
$$

by using (3.2). Also, we have

$$
I_{2} \leqq 2 C_{x, \tau} \lambda \sum_{p=0}^{n-1}\left\|F\left(J_{\lambda}^{n k} x-J_{h}^{k} x\right)-F\left(J_{\lambda}^{n(k-1)+p+1} x-J_{h}^{k} x\right)\right\| .
$$

Employing the uniform continuity of $F$ on bounded sets, we can find a function $\mathscr{E}(h) \equiv \mathscr{E}(h ; x, \tau)$ such that $\mathscr{E}(h) \rightarrow 0$ as $h \rightarrow 0+$ and such that

$$
\sup _{n \lambda \leqq h ; h k \leqq \tau}\left\|F\left(J_{\lambda}^{n k} x-J_{h}^{k} x\right)-F\left(J_{\lambda}^{n(k-1)+p+1} x-J_{h}^{k} x\right)\right\| \leqq \mathscr{E}(h) .
$$

Note that $\left\|J_{\lambda}^{n(k-1)+p+1} x-J_{\lambda}^{n k} x\right\| \leqq C_{x, \tau} h$. Also,

$$
I_{3} \leqq|n \lambda-h|\left\|A_{h} J_{h}^{k-1} x\right\|\left\|J_{\lambda}^{n k} x-J_{h}^{k} x\right\| .
$$

Consequently,

$$
\begin{aligned}
& \left\|J_{\lambda}^{n m} x-J_{h}^{m} x\right\|^{2}=\sum_{k=1}^{m}\left\{\left\|J_{\lambda}^{n k} x-J_{h}^{k} x\right\|^{2}-\left\|J_{\lambda}^{n(k-1)} x-J_{h}^{k-1} x\right\|^{2}\right\} \\
\leqq & \sum_{k=1}^{m} 2 r e\left\langle\left(J_{\lambda}^{n k} x-J_{h}^{k} x\right)-\left(J_{\lambda}^{n(k-1)} x-J_{h}^{k-1} x\right), F\left(J_{\lambda}^{n k} x-J_{h}^{k} x\right)\right\rangle \\
\leqq & 2 \omega_{B_{x, \tau},} h \sum_{k=1}^{m}\left\|J_{\lambda}^{n k} x-J_{h}^{k} x\right\|^{2}+\psi(\lambda, h),
\end{aligned}
$$


where $\psi(\lambda, h) \equiv$ const $(x, \tau)(h+\mathscr{E}(h)+m|\lambda n-h|)$ and note that

$$
\begin{aligned}
& 2 r e\langle x-y, F(x)\rangle \geqq 2\|x\|^{2}-2\|x\|\|y\| \\
= & \|x\|^{2}-\|y\|^{2}+(\|x\|-\|y\|)^{2} \geqq\|x\|^{2}-\|y\|^{2} .
\end{aligned}
$$

Hence, for each $t \in[0, \tau]$, we can write

$$
\left\|J_{\lambda}^{n[t / h]} x-J_{h}^{[t / h]} x\right\|^{2} \leqq 2 \omega_{B_{x, \tau}} \int_{0}^{t}\left\|J_{\lambda}^{n[s / h]} x-J_{h}^{[s / h]} x\right\|^{2} d s+\psi(\lambda, h) .
$$

This is a Gronwall type inequality, and so, we have that

$$
\left\|J_{\lambda}^{n[t / h]} x-J_{h}^{[t / h]} x\right\|^{2} \leqq \psi(\lambda, h) \exp \left(2 \omega_{B_{x, \tau}} t\right) .
$$

Therefore, we have

$$
\begin{aligned}
& \left\|J_{\lambda}^{[t / / \lambda]} x-J_{h}^{[t / h]} x\right\| \\
\leqq & \left\|J_{\lambda}^{[t / \lambda]} x-J_{\lambda}^{n[t / h]} x\right\|+\sqrt{\psi(\lambda, h)} \exp \left(\omega_{B x, \tau} \tau\right) .
\end{aligned}
$$

First, take $\lambda=\varepsilon_{\mu}=2^{-\mu}, h=\varepsilon_{\nu}=2^{-\nu}, m=\left[t / \varepsilon_{\nu}\right]$ and $n=2^{\mu-\nu}$. In this case $\psi\left(\varepsilon_{\mu}, \varepsilon_{\nu}\right)=$ const $(x, \tau)\left(\varepsilon_{\nu}+\mathscr{E}\left(\varepsilon_{\nu}\right)\right) \rightarrow 0$ as $\nu \rightarrow \infty$, and $\left|\left[t / \varepsilon_{\mu}\right]-2^{\mu-\nu}\left[t / \varepsilon_{\nu}\right]\right| \leqq 2^{\mu-\nu}$. So, we see that (by (3.2))

$$
\left\|J_{\varepsilon_{\mu}}^{\left[t / \varepsilon_{\mu}\right]} x-J_{\varepsilon_{\mu}}^{2 \mu-\nu\left[t / \varepsilon_{\nu}\right]} x\right\|=0\left(\varepsilon_{\nu}\right)
$$

and hence

$$
\left\|J_{\varepsilon_{\mu}}^{\left[t / \varepsilon_{\mu}\right]} x-J_{\varepsilon_{\nu}}^{\left[t / \varepsilon_{\nu}\right]} x\right\| \leqq 0\left(\varepsilon_{\nu}\right)+\sqrt{\psi\left(\varepsilon_{\mu}, \varepsilon_{\nu}\right)} \exp \left(\omega_{B_{x, \tau}} \tau\right) .
$$

This means that $\left\{J_{\varepsilon_{\nu}}^{\left[t / \varepsilon_{\nu}\right]} x\right\}$ is a Cauchy sequence. We then set

$$
y(t ; x)=\lim _{\nu \rightarrow \infty} J_{\varepsilon_{\nu}}^{\left[t / \varepsilon_{\nu}\right]} x, \quad t \in[0, \tau],
$$

Finally, we show that the existence of the limit is independent of the sequence chosen. Let $0 \leqq t<\tau$, and $0<\lambda \leqq h<\min \left\{\lambda_{0}, \tau-t\right\}$. Taking, this time $m=[t / h]+1$ and $n=[[t / \lambda] /[t / h]+1]$ we observe that

$$
\left\{\begin{array}{l}
m h \leqq t+h, n \lambda \leqq h,|t-n \lambda m| \leqq 2 \lambda+\tau \lambda / h \\
|[t / \lambda]-n m| \lambda \leqq 3 \lambda+\tau \lambda / h, m|n \lambda-h| \leqq 2 h+2 \lambda+\tau \lambda / h
\end{array}\right.
$$

Similarly, as above, taking $\lambda=\varepsilon_{\nu}$, then letting $\nu \rightarrow \infty$, we see using (3.4) and (3.5) that

$$
\left\|y(t ; x)-J_{h}^{[t / h]} x\right\| \leqq \text { const }(x, \tau) \sqrt{3 h+\varepsilon(h)} .
$$

Lemma 3.2. Let $A$ be a D-operator in $X$ satisfying $(I),(R)$ and $(E)$.

(a) For every bounded set $B$ in $D(A)$ and $\tau>0$, there exists $a$ number $\omega_{B, \tau} \geqq 0$ such that 


$$
\left\|y\left(t ; x_{1}\right)-y\left(t ; x_{2}\right)\right\| \leqq \exp \left(\omega_{B, \tau} t\right)\left\|x_{1}-x_{2}\right\|
$$

for $t \in[0, \tau]$ and $x_{1}, x_{2} \in B$.

(b) For every $x \in D(A)$ and $\tau_{0}>0$, there exists a number $\omega_{x, \tau} \geqq 0$ such that

$$
\left\|y(t ; x)-y\left(t^{\prime} ; x\right)\right\| \leqq\left|t-t^{\prime}\right| \exp \left(\omega_{x, \tau} \tau\right)|\| A x| \mid
$$

for $t, t^{\prime} \in[0, \tau]$.

Proof. (a) Let $B$ be a bounded set in $D(A)$ and $\tau_{0}>0$. Take $x_{1}, x_{2} \in B$, then by Proposition 1.3 we have that

$$
\left\|J_{h}^{[t / h]} x_{1}-J_{h}^{[t / h]} x_{2}\right\| \leqq\left(1-h \omega_{B, \tau}\right)^{-[t / h]}\left\|x_{1}-x_{2}\right\|
$$

for some $\omega_{B, \tau} \geqq 0$ and $h$ sufficiently small. Now letting $h \rightarrow+0$, we obtain (a).

(b) Let $x \in D(A), \tau>0$ and set

$$
\begin{aligned}
B_{x, \tau}= & \left\{J_{h}^{m} x ; h \in\left(0, \lambda_{0}\right), m h \in[0, \tau]\right\} \\
& \cup\left\{x-h y ; h \in\left(0, \lambda_{0}\right), y \in A x,\|y\| \leqq\|A x\|+1\right\} .
\end{aligned}
$$

Then, $B_{x, \tau}$ is a bounded set by $(\mathrm{E})$. Now, let $\omega_{x, \tau}$ be a constant associated with this bounded set in the sense of Proposition 1.3 and let $0 \leqq t^{\prime}<t \leqq \tau$. Then, by (3.2),

$$
\begin{aligned}
\left\|J_{h}^{[t / h]} x-J_{h}^{\left[t^{\prime} / h\right]} x\right\| & \leqq \sum_{j=\left[t^{\prime} / h\right]}^{[t / h]-1}\left\|J_{h}^{j+1} x-J_{h}^{j} x\right\| \leqq h \sum_{j=\left[t^{\prime} / h\right]}^{[t / h]-1}\left\|A_{h} J_{h}^{j} x\right\| \\
& \leqq\left|[t / h]-\left[t^{\prime} / h\right]\right| h\left(1-h \omega_{x, \tau}\right)^{-[t / h]}\|A x\| .
\end{aligned}
$$

Letting $h \rightarrow+0$, we have (b).

Consequently, we have the following main theorem:

THEOREM 3.1 If $A$ is a D-operator in $X$ satisfying $(I),(R)$ and $(E)$. Then there exists a semi-group $\{T(t)\}$ on $\overline{D(A)}$ such that

$$
T(t) x=\lim _{\lambda \rightarrow+0}(I-\lambda A)^{-[t / \lambda]} x \text { for } t \geqq 0 \text { and } x \in D(A),
$$

and the convergence is uniform with respect to $t$ in every finite interval.

Proof. In view of Lemma 3.1, set $T(t) x=y(t ; x)$ for $t \geqq 0$ and $x \in D(A)$. First, by using Lemma 3.2 (a), we can obtain a unique extension of $T(t)$ to $\overline{D(A)}$ by continuity, we denote this extension by the same symbol $T(t)$. Then each $T(t)$ maps $\overline{D(A)}$ into itself, and also for every bounded set $B$ in $\overline{D(A)}$ and $\tau>0$ there exists a number $\omega_{B,-} \geqq 0$ such that 


$$
\|T(t)\|_{\operatorname{Lip}(B)} \leqq \exp \left(\omega_{B, \tau} t\right), \quad t \in[0, \tau] .
$$

To establish the semi-group property, first take $x \in D(A)$ and $t, s \geqq 0$ with $t+s \leqq \tau$. Let $B_{x, \tau}$ be the bounded set defined in the proof of Lemma 3.1 and $N(T(s) x)$ be a bounded neighborhood of $T(s) x$ (small), and then consider the bounded set $\overline{B_{x, \tau}} \cup N(T(s) x)$. Now using Proposition 1.3 and (3.8), it is seen that $\|T(t+s) x-T(t) T(s) x\|$ can be made arbitrarily small. (3.7) was established in Lemma 3.1.

REMARK 3.1. In Theorem 3.1, (3.7) holds for $x \in \overline{D(A)}$, if either of the following conditions is satisfied:

$$
R(I-\lambda A) \supset \overline{D(A)} \text { for } \lambda \in\left(0, \lambda_{0}\right), \quad \text { or }
$$

$A$ is closed.

In fact, if (3.9) holds, then by Proposition $1.3\left\{J_{\lambda}^{[t / \lambda]\}}\right.$ is equi-Lipschitz continuous on bounded sets in $\overline{D(A)}$. Hence, Lemma 3.1 implies the convergence (3.7) for all $x \in \overline{D(A)}$. Next, assume that $A$ is closed. Let $x \in \overline{D(A)}, t \in[0, \tau]$, and then choose a sequence $\left\{x_{n}\right\} \subset D(A)$ with $x_{n} \rightarrow x$. Let $B=\overline{\left\{x_{n}\right\}}$, then by Proposition 1.2, we see that there is a number $\lambda_{B}$ such that if $\lambda \in\left(0, \lambda_{B}\right)$, then $y_{n}(\lambda)=J_{\lambda} x_{n} \rightarrow v_{\lambda} \in X$. Hence, $A y_{n}(\lambda) \ni \lambda^{-1}\left(y_{n}(\lambda)-x_{n}\right) \rightarrow \lambda^{-1}\left(v_{\lambda}-x\right)$. This means that

$$
\lambda^{-1}\left(v_{\lambda}-x\right) \in A v_{\lambda} \text {, i.e., } x \in(I-\lambda A) v_{\lambda} \subset R(I-\lambda A) \text {. }
$$

Therefore, Proposition 1.3 implies that $\left\{J_{\lambda}^{[t / \lambda]}\right\}$ is equi-Lipschitz continuous on $B$, and so, Lemma 3.1 implies the convergence (3.7) for the $x$.

4. Differentiability of the Constructed Semi-Groups. The differentiability of the semi-group obtained by Theorem 3.1 is investigated in this section. The central part of the arguments is based on the results of Kato [4] and [5]. Throughout this section $X$ is assumed to have a uniformly convex dual.

Let $A$ be a $D$-operator in $X$ satisfying $(I),(R)$ and $(E)$. Set $\varepsilon_{n}=2^{-n}$ and $I_{r}=[0, r]$ for $r=1,2,3, \cdots$ and define $J_{n}=\left(I-\varepsilon_{n} A\right)^{-1}$ and $A_{n}=\varepsilon_{n}^{-1}\left[J_{n}-I\right]$ for $n$ with $\varepsilon_{n} \in\left(0, \lambda_{0}\right)$.

In view of (3.2), we note that for each $r$,

$$
\left\|A_{n} J_{n}^{\left[t / \varepsilon_{n}\right]} x\right\| \leqq\left(1-\varepsilon_{n} \omega_{B_{x, r}}\right)^{-\left[t / \varepsilon_{n}\right]-1} \mid\|A x\| \|,
$$

$n$ sufficiently large and $t \in[0, r]$, for the bounded set

$$
\begin{aligned}
B_{x, r}= & \left\{J_{n}^{\left[t / \varepsilon_{n}\right]} x ; t \in[0, r], n \text { sufficiently large }\right\} \\
& \cup\left\{x-\varepsilon_{n} y ; n \text { large, } y \in A x,\|y\| \leqq\|A x\|+1\right\} .
\end{aligned}
$$


Since

$$
\begin{aligned}
\left(1-\varepsilon_{n} \omega_{B x, r}\right)^{-1} & =1+\omega_{B_{x, r}} \varepsilon_{n}\left(1-\varepsilon_{n} \omega_{B_{x, r}}\right)^{-1} \\
& \leqq \exp \left(\omega_{B_{x, r}} \varepsilon_{n}\left(1-\varepsilon_{n} \omega_{B_{x, r}}\right)^{-1}\right)
\end{aligned}
$$

for $n$ sufficiently large and $t \in[0, r]$. Hence,

$$
\left\|A_{n} J_{n}^{\left[t / \varepsilon_{n}\right]} x\right\| \leqq \exp \left(\omega_{B_{x, r}}\left(r+\varepsilon_{n}\right)\left(1-\varepsilon_{n} \omega_{B_{x, r}}\right)^{-1}\right)\|A x\|
$$

for $n$ sufficiently large and $t \in[0, r]$. Therefore, if we set $f_{n}(t ; x)=$ $A_{n} J_{n}^{\left[t / \varepsilon_{n}\right]} x$ for $t \geqq 0$ and $x \in D(A)$, then $f_{n}(t ; x) \in A J_{n}^{\left[t / \varepsilon_{n}\right]+1} x$, and

(4.1) for every $r,\left\|f_{n}(t ; x)\right\|$ is uniformly bounded with respect to $n$ sufficiently large and $t \in[0, r]$.

Also, since

$$
\begin{aligned}
\int_{0}^{\left[t / \varepsilon_{n}\right] \varepsilon_{n}} A_{n} J_{n}^{\left[s / \varepsilon_{n}\right]} x d s & =\varepsilon_{n} \sum_{k=1}^{\left[t / \varepsilon_{n}\right]} A_{n} J_{n}^{(k-1)} x \\
& =\left[J_{n}^{\left[t / \varepsilon_{n}\right]}-I\right] J_{n} x+\varepsilon_{n}\left\{A_{n} x-A_{n} J_{n}^{\left[t / \varepsilon_{n}\right]} x\right\},
\end{aligned}
$$

we have

$$
\left\|\left[J_{n}^{\left[t / \varepsilon_{n}\right]}-I\right] J_{n} x-\int_{0}^{t} f_{n}(s ; x) d s\right\|=O\left(\varepsilon_{n}\right) .
$$

The main result of this section is the following:

THEOREM 4.1. Let $A$ be a demi-closed D-operator satisfying $(I)$, $(R)$ and $(E)$, and $\{T(t)\}$ be the semi-group on $\overline{D(A)}$ obtained by Theorem 3.1. Then for $x \in D(A)$,

(i) $T(t) x \in D(A)$ for $t \geqq 0$,

(ii) there exists a function $f(\cdot ; x)$ on $[0, \infty)$ such that

$$
f(t ; x) \in \hat{A} T(t) x
$$

for almost all $t \geqq 0$, where $\hat{A} x=\{y \in \overline{c o} A x ;\|y\| \leqq\|A x\| \mid\}$, and

$$
T(t) x-x=\int_{0}^{t} f(s ; x) d s
$$

Proof. Take $x \in D(A)$ and $p$ with $1<p<+\infty$. Set $f_{n}(t ; x)=$ $A_{n} J_{n}^{\left[t / \epsilon_{n}\right]} x$, then by (4.1) $\left\{\left.f_{n}(\cdot ; x)\right|_{I_{r}} ; n\right.$ sufficiently large $\}$ forms a bounded set of $L^{p}\left(I_{r} ; X\right)$ for integer $r$. Thus by moving $r$ and using the diagonal process, we find a subsequence $\{q\} \subset\{n\}$ and a function $f(\cdot ; x)$ on $[0, \infty)$ such that $\left.f_{q}(\cdot ; x)\right|_{I_{r}}$ converges weakly to $\left.f(\cdot ; x)\right|_{I_{r}}$ in $L^{p}\left(I_{r} ; X\right)$ for each integer $r$. Hence,

$$
x^{*} \int_{0}^{t} f_{q}(s ; x) d s \longrightarrow x^{*} \int_{0}^{t} f(s ; x) d s
$$

for all $x^{*} \in X^{*}$ and $t \geqq 0$. Thus (4.3) follows from (4.2). Write $V(t)$ 
for the set of all weak cluster points of $\left\{f_{n}(t ; x) ; n\right\}$ for $t \geqq 0$, then Lemma 3.1 and Proposition 1.7 (a) imply that $T(t) x \in D(A), V(t) \neq \varnothing$, and $V(t) \subset A T(t) x$ for $t \geqq 0$. Hence, by the same argument as in Kato [5; Lemma 8.2] we see that $f(t ; x) \in \overline{c o} A T(t) x$ for almost all $t \geqq 0$. And, in a similar way to Kato [5; Lemma 6.2], $\|f(t ; x)\| \leqq$ || $A T(t) x \mid \|$ for almost all $t \geqq 0$. Thus, it follows that $f(t ; x) \in \hat{A} T(t) x$ for almost all $t \geqq 0$.

REMARK 4.1. Let $A$ be a demi-closed $D$-operator in $X$ satisfying $(I),(R)$ and $(E)$, and $\{T(t)\}$ be the semi-group obtained by Theorem 3.1 , then $\left\{\left.T(t)\right|_{D(A)} ; t \geqq 0\right\}$ forms a semi-group on $D(A)$ by the above theorem. By (4.3), we see that the infinitesimal generator $A_{0}$ of $\left\{\left.T(t)\right|_{D(A)}\right\}$ is densely defined in $D(A)$.

In view of these results and Proposition 1.6, we have the following.

Theorem 4.2. Let $A$ be a maximal $\left(D,\left\{\omega_{n}\right\}\right)$-operator on $\overline{D(A)}$ satisfying $(I),(R)$ and $(E)$. Then there is a uniquely determined semi-group $\{T(t)\}$ on $D(A)$ such that for each $x \in D(A)$,

$(d / d t) T(t) x \in A^{0} T(t) x \quad$ for almost all $t \geqq 0$.

Theorem 4.3. If $A$ is a single-valued, demi-closed D-operator in $X$ satisfying $(I),(R)$ and $(E)$. Then there is a uniquely determined semi-group $\{T(t)\}$ on $D(A)$ such that

(a) for $x \in D(A), A T(t) x$ is weakly continuous in $t \geqq 0$ and

$$
T(t) x-x=\int_{0}^{t} A T(s) x d s \quad \text { for } t \geqq 0,
$$

(b) $A$ is the weak infinitesimal generator and the infinitesimal generator $A_{0}$ is densely defined in $D(A)$.

Proof. Using the notation in the proof of Theorem 4.1, we have that $V(t)$ is a singleton, since $A$ is single-valued. And thus, by Proposition 1.7, $w$-lim $f_{n}(t ; x)=A T(t) x$ for $t \geqq 0$. The strong continuity of $T(t) x$ and the boundedness of $A T(t) x$ give that $A T(t) x$ is weakly continuous in $t \geqq 0$. Finally (4.4) follows directly from (4.2).

CoRollary 4.1. If $A$ is a demi-closed $D$-operator in $X$ satisfying $(I),(R)$ and $(E)$, and $A^{0}$ is single-valued, then there exists a unique semi-group $\{T(t)\}$ on $D(A)$ such that for $x \in D(A),(d / d t) T(t) x=A^{0} T(t) x$ for almost all $t \geqq 0$. 
Proof. In this case, note that we have that $\hat{A} \equiv A^{0}$, where $\hat{A}$ is defined in Theorem 4.1 by $\hat{A} x=\{y \in \overline{c o} A x ;\|y\|=\|A x\|\}$.

CoROllary 4.2. If $A$ is a demi-closed D-operator in $X$ satisfy$\operatorname{ing}(I)$ and $(R)$ and $\left\|J_{2} x\right\| \leqq(1+M \lambda)\|x\|+N \lambda$ for $\lambda \in\left(0, \lambda_{0}\right), x \in R_{\lambda}$, where $M$ and $N$ are nonnegative, then there is a semi-group $\{T(t)\}$ on $D(A)$ such that $(d / d t) T(t) x \in \overline{c o} A T(t) x$ for almost all $t \geqq 0$ and $\|T(t) x\| \leqq e^{M t}(\|x\|+N t)$ for $t \geqq 0$.

Proof. By Proposition 1.4, $A$ satisfies condition $(E)$ and also we have that $\left\|J_{\lambda}^{[t / \lambda]} x\right\| \leqq(1+M \lambda)^{[t / \lambda]}(\|x\|+N t)$, hence using Theorem 4.1 we have the assertion.

Corollary 4.3. If $A$ is a single-valued, demi-closed D-operator in $X$ satisfying $(I)$ and $(R)$ and $\sup \|A x\|=N<+\infty$, then $A$ is the weak infinitesimal generator of a semi-group $\{T(t)\}$ on $D(A)$ such that $\|T(t) x\| \leqq\|x\|+N t$ for $t \geqq 0$ and $x \in D(A)$ and

$$
\sup \{\|A T(t) x\| ; t \geqq 0, x \in D(A)\} \leqq N \text {. }
$$

Proof. Employ Proposition 1.4.

In the remainder of this section, we consider the case in which $X$ is uniformly convex.

Lemma 4.1. Let $A$ be a demi-closed D-operator in $X$ satisfying $(I),(R)$ and $(E)$ such that $A^{0}$ is a single-valued operator with $D\left(A^{0}\right)=D(A)$. Then if $\{T(t)\}$ is the semi-group on $D(A)$ obtained by Theorem 4.1, we have for $x \in D(A)$,

(a) $\||A T(t) x \||$ is of bounded variation on every finite interval and has no positive jumps,

(b) the right derivative $D^{+} T(t) x$ exists and is strongly rightcontinuous in $t$, and $D^{+} T(t) x=A^{0} T(t) x$ for $t \geqq 0$,

(c) $A^{0} T(t) x$ is strongly continuous except possibly at a c countable number of points $t$.

Proof. (a) Take $x \in D(A)$. Then by the same argument as in Kato [5; Lemma 6.6] we obtain that

$$
e^{-\omega_{B, \tau^{t}}}|||A T(t) x|\left\|\leqq e^{-\omega_{B, \tau} t}|| A T(r) x \mid\right\|
$$

for all $r$ and $t$ with $0 \leqq r \leqq t \leqq \tau$. Thus, $\|A A T(t) x\|$ is of bounded variation.

(b) Take $x \in D(A)$ and $t \geqq 0$. Choose a sequence $t_{k} \downarrow t$. Then by the proof of Kato [5; Theorem 7.5] we see that $\left\{A^{0} T\left(t_{k}\right) x\right\}$ contains a subsequence which converges strongly to $A^{0} T(t) x$. So, $A^{0} T(t) x$ is strongly 
right-continuous in $t$. But, since $T(t) x-x=\int_{0}^{t} A^{0} T(s) x d s$ by Theorem 4.1, it follows that $D^{+} T(t) x=A^{0} T(t) x$ for each $t$.

(c) By (a) $\left\|A^{0} T(t) x\right\|=\|A T(t) x\|$ is continuous except for a countable number of points $t$. In order to show that $A^{0} T(t) x$ is continuous except for those points, it suffices to repeat the same argument as in (b) with $t_{k} \uparrow t$. But the continuity at $t$ of $\left\|A^{0} T(t) x\right\|$ assures that $\lim _{k}\left\|A^{0} T\left(t_{k}\right) x\right\|=\left\|A^{0} T(t) x\right\|$. Thus the uniform convexity implies that $A^{0} T(t) x$ is strongly continuous at the $t$.

Consequently, we have the following:

Theorem 4.4. Let $X$ be uniformly convex. If $A$ is a demiclosed D-operator in $X$ satisfying $(I),(R)$ and $(E)$ such that $A^{0}$ is a single-valued operator with $D\left(A^{\circ}\right)=D(A)$, then $A^{0}$ is the infinitesimal generator of a unigue semi-group $\{T(t)\}$ on $D(A)$ such that for $x \in D(A), D^{+} T(t) x=A^{0} T(t) x$ for $t \geqq 0$, and $D^{+} T(t)$ is strongly rightcontinuous in $t \geqq 0$.

The following results are the direct consequences of the above theorem.

Corollary 4.4. Let $X$ be uniformly convex. If $A$ satisfies the assumptions of Theorem 4.2, then $A^{0}$ is the infinitesimal generator of a unique semi-group $\{T(t)\}$ on $D(A)$ such that for $x \in D(A), T(t) x$ is strongly right-continuously differentiable in $t$ and $D^{+} T(t) x=A^{0} T(t) x$ for $t \geqq 0$.

CoRollary 4.5. Let $X$ be uniformly convex. If $A$ is a singlevalued, demi-closed $D$-operator in $X$ satisfying $(I),(R)$ and $(E)$, then $A$ is the infinitesimal generator of a unique semi-group $\{T(t)\}$ on $D(A)$ such that for $x \in D(A), T(t) x$ is strongly right-differentiable in $t$ and $D^{+} T(t) x=A T(t) x$ for each $t \geqq 0$.

REMARK 4.2. Let $X$ be uniformly convex. If $A$ is a closed dissipative operator in $X$ satisfying $(R)$, then $A^{0}$ is the infinitesimal generator of a unique semi-group $\{T(t)\}$ of contractions on $D(A)$ such that for $x \in D(A), T(t) x$ is strongly right-continuously differentiable in $t$ and $D^{+} T(t) x=A^{0} T(t) x$ for $t \geqq 0$. For details, see [10].

\section{APPENDIX}

A.1. After this paper was submitted for publication, Crandall and Liggett gave (in "Generation of semigroups of nonlinear trans- 
formations on general Banach spaces", to appear) a new method for constructing a semigroup of nonlinear contractions in a general Banach space. The main results in their paper can be extended straightforwardly to our case. As was stated in $\S 1$, Propositions 1.2 and 1.3 are valid for general Banach spaces. Using these propositions in a similar way to their proof, we can obtain the assertion of Theorem 3.1, without assuming that $X^{*}$ is uniformly convex. Also, we can obtain a similar result to theirs on the differentiability of semigroups of nonlinear contractions. For details, we shall publish elsewhere.

A.2. We did not give in the body of this paper any examples of $D$-operator satisfying conditions $(I),(R)$ and $(E)$. We state here a simple example of a $D$-operator which is not necessarily a dissipative operator.

Let $\Omega$ be a bounded domain with smooth boundary in $R^{N}$ and let us consider the Cauchy problem

$$
\begin{aligned}
& (\partial / \partial t) u_{1}=\Delta u_{1}+\Phi u_{2}, \\
& (\partial / \partial t) u_{2}=\Delta u_{2}
\end{aligned}
$$

with the initial condition

$$
\begin{aligned}
& u_{1}(0, s)=u_{1}(s), \\
& u_{2}(0, s)=u_{2}(s),
\end{aligned}
$$

over the Hilbert space $H=L_{2}(\Omega) \times L_{2}(\Omega)$ with the inner product

$$
\langle u, v\rangle=\left\langle u_{1}, v_{1}\right\rangle+\left\langle u_{2}, v_{2}\right\rangle, u=\left(\begin{array}{l}
u_{1} \\
u_{2}
\end{array}\right), v=\left(\begin{array}{l}
v_{1} \\
v_{2}
\end{array}\right) \text {. }
$$

It is well-known that the operator $\Delta$ with domain $H^{2}(\Omega) \cap H_{0}^{1}(\Omega)$ is $m$ dissipative. We then assume that the operator $\Phi$ is locally bounded on $X$ and Lipschitz continuous on bounded sets.

Now, let us define an operator $A$ in $H$ by the relation

$$
A u=\left(\begin{array}{l}
\Delta u_{1}+\Phi u_{2} \\
\Delta u_{2}
\end{array}\right) \text { for } u \in D(A)=\left\{u=\left(\begin{array}{l}
u_{1} \\
u_{2}
\end{array}\right) ; u_{1}, u_{2} \in H^{2}(\Omega) \cap H_{0}^{1}(\Omega)\right\} .
$$

Then the problem (A.1) is understood as the $A C P$ for $A$ in the space $H$.

In the following, we demonstrate that $A$ so defined is a demiclosed $D$-operator satisfying conditions $(I),(R)$ and $(E)$.

(a) Let $B$ be any bounded set in $H$ and $u, v \in B \cap D(A)$. Then 


$$
\begin{aligned}
& \langle A u-A v, u-v\rangle \\
= & \left\langle\Delta\left(u_{1}-v_{1}\right), u_{1}-v_{1}\right\rangle+\left\langle\Phi u_{2}-\Phi v_{2}, u_{1}-v_{1}\right\rangle \\
& +\left\langle\Delta\left(u_{2}-v_{2}\right), u_{2}-v_{2}\right\rangle \\
\leqq & -\left\|u_{1}-v_{1}\right\|_{1}^{2}-\left\|u_{2}-v_{2}\right\|_{1}^{2}+\left\|\Phi u_{2}-\Phi v_{2}\right\|\left\|u_{1}-v_{1}\right\| \\
\leqq & \gamma_{B}\left\|u_{2}-v_{2}\right\|\left\|u_{1}-v_{1}\right\| \leqq \gamma_{B} / 2 \cdot\left(\left\|u_{1}-v_{1}\right\|^{2}+\left\|u_{2}-v_{2}\right\|^{2}\right) \\
\leqq & \omega_{B}\|u-v\|^{2},
\end{aligned}
$$

where $\gamma_{B}$ is the smallest Lipschitz constant of $\Phi$ on the bounded set $B$. Hence, $A$ is a $D$-operator.

(b) Let $v \in X, \lambda>0$ and let us consider the equation

$$
u-\lambda A u=v,
$$

or equivalently,

$$
\left\{\begin{aligned}
(I-\lambda \Delta) u_{1}-\lambda \Phi u_{2} & =v_{1}, \\
(I-\lambda \Delta) u_{2} & =v_{2},
\end{aligned}\right.
$$

Since $\Delta$ is $m$-dissipative, we obtain a unique solution

$$
u_{2}=(I-\lambda \Delta)^{-1} v_{2}
$$

of the second equation of (A.3). Substituting this into the first equation and using the $m$-dissipativity of $\Delta$, we get

$$
u_{1}=(I-\lambda \Delta)^{-1}\left[v_{1}+\lambda \Phi(I-\lambda \Delta)^{-1} v_{2}\right] \text {. }
$$

Therefore, $u=\left(\begin{array}{l}u_{1} \\ u_{2}\end{array}\right)$ is the unique solution of (A.2) and since $\lambda>0$ and $v \in X$ were arbitrary, we see that $I-\lambda A$ is injective and $R(I-\lambda A)=H$ for all $\lambda>0$. Hence, $A$ satisfies $(I)$ and $(R)$.

(c) From (A.4) and (A.5) it follows that

$$
\begin{aligned}
& \|u\|^{2}=\left\|u_{1}\right\|^{2}+\left\|u_{2}\right\|^{2} \\
\leqq & \left\|v_{1}\right\|^{2}+2 \lambda\left\|v_{1}\right\|\left\|\Phi(I-\lambda \Delta)^{-1} v_{2}\right\|+\lambda^{2}\left\|\Phi(I-\lambda \Delta)^{-1} v_{2}\right\|^{2}+\left\|v_{2}\right\|^{2} \\
\leqq & \|v\|^{2}+2 \lambda\|v\|\left\|\Phi(I-\lambda \Delta)^{-1} v_{2}\right\|+\lambda^{2}\left\|\Phi(I-\lambda \Delta)^{-1} v_{2}\right\|^{2} \\
= & \left(\|v\|+\lambda\left\|\Phi(I-\lambda \Delta)^{-1} v_{2}\right\|\right)^{2}
\end{aligned}
$$

or

$$
\left\|J_{\lambda} v\right\| \leqq\|v\|+\lambda\left\|\Phi\left(I-\lambda_{\Delta}\right)^{-1} v_{2}\right\|,
$$

where $u=J_{\lambda} v, \lambda>0$ and $v \in X$. Now, let $v \in H, \tau>0, \lambda>0$, and let $n \lambda \in[0, \tau]$, then

$$
\begin{aligned}
\left\|J_{\lambda}^{n} v\right\| & \leqq\left\|J_{\lambda}^{n-1} v\right\|+\lambda\left\|\Phi(I-\lambda \Delta)^{-1}\left[J_{\lambda}^{n-1} v\right]_{2}\right\| \\
& \leqq\left\|J_{\lambda}^{n-2} v\right\|+\lambda\left\{\left\|\Phi(I-\lambda \Delta)^{-1}\left[J_{\lambda}^{n-2} v\right]_{2}\right\|+\left\|\Phi\left(I-\lambda_{\Delta}\right)^{-1}\left[J_{\lambda}^{n-1} v\right]_{2}\right\|\right\}
\end{aligned}
$$

and inductively, 


$$
\leqq\|v\|+\lambda \sum_{i=0}^{n-1}\left\|\Phi(I-\lambda \Delta)^{-1}\left[J_{\lambda}^{i} v\right]_{2}\right\|,
$$

where $\left[J_{\lambda}^{i} v\right]_{2}$ means the second component of $J_{\lambda}^{i} v$. But, since $\left[J_{\lambda}^{i} v\right]_{2}=$ $\left(I-\lambda_{\Delta}\right)^{-i} v_{2}$,

$$
\begin{aligned}
\left\|J_{\lambda}^{n} v\right\| & \leqq\|v\|+\lambda \sum_{i=1}^{n}\left\|\Phi(I-\lambda \Delta)^{-i} v_{2}\right\| \\
& \leqq\|v\|+\tau \sup _{0 \leqq i \lambda \leqq \tau}\left\|\Phi(I-\lambda \Delta)^{-i} v_{2}\right\| .
\end{aligned}
$$

Let $B$ be any bounded set in $H$. Since $\left(I-\lambda_{4}\right)^{-1}$ is a contraction on $H$, the set $\left\{\left(I-\lambda_{4}\right)^{-i} v_{2} ; v \in B, 0 \leqq i \lambda \leqq \tau\right\}$ is bounded in $L_{2}(\Omega)$. On the other hand, $\Phi$ maps bounded sets into bounded sets by assumption, and hence

$$
\sup \left\{\| \Phi\left(\left(I-\lambda_{\triangle}\right)^{-i} v_{2} \| ; v \in B, 0 \leqq i \lambda \leqq \tau\right\}=M_{B, \tau}<+\infty .\right.
$$

Consequently,

$$
\left\|J_{\lambda}^{n} v\right\| \leqq \sup \{\|v\| ; v \in B\}+\tau M_{B, \tau}
$$

for $v \in B, \lambda>0$ and $n \lambda \in[0, \tau]$, which means that $A$ satisfies condition $(E)$.

(d) Finally, we show that $A$ is demi-closed. Assume that $u^{(n)} \in D(A), u^{(n)} \rightarrow u$ and that $A u^{(n)} \rightarrow v$ in $H$. Then, $u_{i}^{(n)} \rightarrow u_{i}, i=1$, 2, $\Phi u_{2}^{(n)} \rightarrow \Phi u_{2}$, and $\Delta u_{2}^{(n)} \rightarrow v_{2}$ in $L_{2}(\Omega)$. Since the closed linear operator $\Delta$ is demi-closed, we have that $v_{2}=\Delta u_{2}$. Also, $\Delta u_{1}^{(n)} \rightarrow v_{1}-\Phi u_{2}$; hence, $v_{1}-\Phi u_{2}=\Delta u_{1}$. Consequently, $v=A u$. This means that $A$ is demi-closed.

From the above, it can be seen that other $D$-operators can be exhibited by replacing the operator $\Delta$ by any $m$-dissipative operator satisfying the assumption of Proposition 1.4 (a). Also, we can consider unbounded operators $\Phi$ by restricting the Hilbert space $H=H_{1} \times H_{2}$ so that $\Phi$ is a locally bounded, locally Lipschitz continuous operator on a Hilbert space $H_{1}$ into another Hilbert space $H_{2}$.

\section{REFERENCES}

1. H. Brezis and A. Pazy, Semi-groups of nonlinear contractions on convex sets, J. Functional Analysis, 6 (1970), 237-281.

2. M. G. Crandall and A. Pazy, Semi.groups of nonlinear contractions and dissipative sets, Jour. Func. Anal., 3 (1969), 376-418.

3. Y. Kōmura, Differentiability of nonlinear semi-groups, Jour. Math. Soc. Japan, 21 (1969), 375-402.

4. T. Kato, Nonlinear semi-groups and evolution equations, Jour. Math. Soc. Japan, 19 (1967), 503-520.

5. — Acretive operators and nonlinear evolution equations in Banach spaces, Proc. Symposium Nonlinear Func. Anal., Amer. Math. Soc., (1970), 138-161. 
6. I. Miyadera, On the convergence of nonlinear semi-groups, Tôhoku Math. Jour., 21 (2), (1969), 221-236.

7. - On the convergence of nonlinear semi-groups, II, Jour. Math. Soc. Japan, 21 (1969), 403-412.

8. I. Miyadera and S. Oharu, Approximation of semi-groups of nonlinear operators, Tôhoku Math. Jour., 22 (1970), 24-47.

9. S. Oharu, Note on the representation of semi-groups of nonlinear operators, Proc. Japan Acad., 42 (1966), 1149-1154.

10. On the generation of semi-groups of nonlinear contractions, J. Math. Soc. Japan, 22 (4), (1970), 526-550.

Received May 25, 1970 and in revised form April 19, 1971.

Georgetown University, Washington, D.C.,

WASEDA UNIVERSITY, TOKYO 


\section{PACIFIC JOURNAL OF MATHEMATICS}

\section{EDITORS}

H. SAMELSON

Stanford University

Stanford, California 94305

C. R. HobBY

University of Washington

Seattle, Washington 98105
J. DugundjI

Department of Mathematics

University of Southern California

Los Angeles, California 90007

RICHARD ARENS

University of California

Los Angeles, California 90024

\section{ASSOCIATE EDITORS}

E. F. BECKENBACH

B. H. NeUMaNN

F. WOLF

K. YoshidA

\section{SUPPORTING INSTITUTIONS}

UNIVERSITY OF BRITISH COLUMBIA

CALIFORNIA INSTITUTE OF TECHNOLOGY

UNIVERSITY OF CALIFORNIA

MONTANA STATE UNIVERSITY

UNIVERSITY OF NEVADA

NEW MEXICO STATE UNIVERSITY

OREGON STATE UNIVERSITY

UNIVERSITY OF OREGON

OSAKA UNIVERSITY
UNIVERSITY OF SOUTHERN CALIFORNIA STANFORD UNIVERSITY

UNIVERSITY OF TOKYO

UNIVERSITY OF UTAH

WASHINGTON STATE UNIVERSITY UNIVERSITY OF WASHINGTON

$\stackrel{*}{*} \stackrel{*}{*} \stackrel{*}{*}$ AMERICAN MATHEMATICAL SOCIETY
NAVAL WEAPONS CENTER 


\section{Pacific Journal of Mathematics}

Vol. 39 , No. 1

May, 1971

Charles A. Akemann, A Gelfand representation theory for $C^{*}$-algebras ....

Sorrell Berman, Spectral theory for a first-order symmetric system of

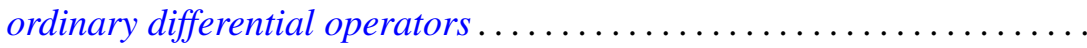

Robert L. Bernhardt, III, On splitting in hereditary torsion theories ........

J. L. Brenner, Geršgorin theorems, regularity theorems, and bounds for determinants of partitioned matrices. II. Some determinantal identities ..........................................

Robert Morgan Brooks, On representing $F^{*}$-algebras .............. 51

Lawrence Gerald Brown, Extensions of topological groups........... 71

Arnold Barry Calica, Reversible homeomorphisms of the real line ........ 79

J. T. Chambers and Shinnosuke Oharu, Semi-groups of local Lipschitzians in

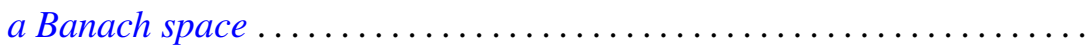

Thomas J. Cheatham, Finite dimensional torsion free rings .............

Byron C. Drachman and David Paul Kraines, A duality between

transpotence elements and Massey products ...................

Richard D. Duncan, Integral representation of excessive functions of a

Markov process ......................................

George A. Elliott, An extension of some results of Takesaki in the reduction

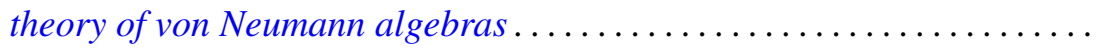

Peter C. Fishburn and Joel Spencer, Directed graphs as unions of partial

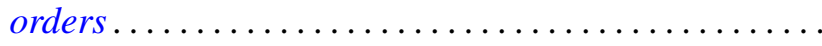

Howard Edwin Gorman, Zero divisors in differential rings ...

Maurice Heins, A note on the Löwner differential equations...

Louis Melvin Herman, Semi-orthogonality in Rickart rings. .

David Jacobson and Kenneth S. Williams, On the solution of linear G.C.D.

equations

Michael Joseph Kallaher, On rank 3 projective planes ... . .

Donald Paul Minassian, On solvable $O^{*}$-groups ...........

Nils Øvrelid, Generators of the maximal ideals of $A(\bar{D})$

Mohan S. Putcha and Julian Weissglass, A semilattice decomposition into

semigroups having at most one idempotent ............

Robert Raphael, Rings of quotients and $\pi$-regularity ....

J. A. Siddiqi, Infinite matrices summing every almost periodic sequence. .

Raymond Earl Smithson, Uniform convergence for multifunctions ...

Thomas Paul Whaley, Mulitplicity type and congruence relations in

universal algebras...

Roger Allen Wiegand, Globalization theorems for locally finitely generated modules... 